\title{
José Domínguez Caparrós: El moderno endecasílabo dactíli- co, anapéstico o de gaita gallega. Sevilla: Rhythmica. Revista española de métrica comparada. Anejo III, 2010.
}

El catedrático de Teoría de la Literatura y Literatura comparada José Domínguez Caparrós aborda en este libro un metro relativamente descuidado hasta ahora, o al menos con una presencia menor en los estudios de versificación española, con lo que da un nuevo paso en su ya dilatada y fructífera trayectoria en la investigación sobre el arte de la métrica, ámbito en el que ha publicado numerosas y fundamentales obras, tanto de carácter general y teórico como histórico y concreto, y que comienza con su imprescindible Contribución a la historia de las teorías métricas en los siglos XVIII y XIX, de 1974, para seguir con títulos como Elementos de métrica española (2005), Métrica española (1993), Estudios de métrica (1999), Nuevos estudios de métrica (2007), Métrica comparada (1994), Métrica de Cervantes (2002), Métrica y poética. Bases para la fundamentación de la métrica en la teoría literaria moderna (1987), el Diccionario de métrica española (1999) o la Contribución a la bibliografía de los últimos treinta años sobre la métrica española (1987).

Como es habitual en el autor, Domínguez Caparrós basa todo el estudio en una profusa y completa documentación que recopila y analiza con rigor y exhaustividad cuanto se ha dicho de interés sobre este tipo de endecasílabo, desde la primera descripción sistemática que hace Manuel Milá y Fontanals en su artículo «Del decasílabo y endecasílabo anapésticos» de 1875 -0 , más aún, desde las menciones más o menos ocasionales que de él hacen Tomás de Iriarte, Sinibaldo de Mas, José Coll y Vehí o Juan Francisco Masdeu- hasta las reflexiones y noticias al respecto de Tomás Navarro Tomás, uno de los últimos metricistas 
de la modernidad en interesarse por este verso. Entre medias, se destaca la poderosa irrupción de Rubén Darío, responsable de la reintroducción y canonización del endecasílabo dactílico, de su difusión en el modernismo $\mathrm{y}$, en último caso, de la aparición de las consiguientes polémicas, como la disputa sabrosa y afilada que mantienen Eduardo de la Barra y Leopoldo Alas Clarín, de la que Domínguez Caparrós da cumplida referencia y detallado análisis.

En cuanto a la estructura del estudio, aunque son cinco las divisiones formales y explícitas - contando conclusión y bibliografia-, la obra está dividida en esencia en dos partes bien diferenciadas que se complementan y refuerzan mutuamente. La primera es la más breve pero a la vez la más densa, puesto que en ella se concentra la teoría sobre el endecasílabo dactílico, así como un repaso esquemático a su presencia en la literatura hispánica a lo largo de la historia, tal como ha ido siendo descrita por los principales metricistas y los propios poetas.

La segunda parte, que prácticamente duplica en extensión a la primera, es una revisión crítica de los ejemplos más significativos del uso del endecasílabo dactílico que cabe encontrar en la poesía española culta. Hay en esta sección del libro, sin embargo, una nueva división que toma como referencia y bisagra la figura de Rubén Darío, poeta axial por su cronología y por su importancia, como se ha dicho, en la canonización del metro. De este modo, se suceden los comentarios, siempre con el ritmo y los elementos estróficos como referente principal, de los textos de más de una treintena de autores, siguiendo un orden cronológico -tomando como referencia la fecha de escritura o publicación de los poemas analizados, antes que la de nacimiento de los poetas- que empieza con los poco conocidos Joseph Blanch, José Bassa y Pedro de Peralta Barnuevo y termina con Francisco Vighi, Antonio Carvajal y Pablo Jauralde Pou; esto es, desde mediado el siglo XVII hasta, exactamente, el año 2000. Puede decirse, dada la profusión de textos analizados y el hecho de que con frecuencia se consignan de manera íntegra, que el libro de Domínguez Caparrós funciona también, en cierto modo, como antología. Una antología que no sería temática ni generacional, sino formal, rítmica, y que en su variedad incluye textos de muy 
diversa valía e incluso naturaleza y procedencia, como la letra de la canción «Yolanda», de Pablo Milanés.

El libro comienza con una atinada y pertinente reflexión por parte del autor:

Un verso se constituye como tal, en su individualidad rítmica, cuando su modelo es conscientemente utilizado en un poema. Se necesita, pues, una conciencia de sus rasgos y una manifestación intencionada de los mismos en un poema. Otra cuestión es que esta forma consiga la consagración canónica en el repertorio de formas prestigiosas de la poesía.

De ahí que Domínguez Caparrós insista, a lo largo de la obra, en la diferencia entre endecasílabos dactílicos casuales, de los que es posible encontrar bastantes muestras de una considerable antigüedad, siempre intercalados en composiciones que contienen distintos metros o variados ritmos, y endecasílabos dactílicos independientes, que de manera consciente, y a partir del siglo XVIII, se sitúan en la base de la construcción de estrofas o composiciones articuladas en torno a su carácter dactílico.

Como subraya el autor a continuación, las cuestiones formales no pueden separarse en estos casos de las históricas y las estéticas, por lo que el éxito de un metro y su consolidación dependen, como es lógico, del uso más o menos afortunado que de él se haga. Son las composiciones que lo utilizan las que determinan su lugar en la tradición, y de ahí la necesidad de destacar el papel que juega Rubén Darío, que es quien «canoniza el verso endecasílabo acentuado en $4^{\mathrm{a}}$ y $7^{\mathrm{a}}$ sílabas, y frecuentemente también en la $1^{\mathrm{a}}$ »». Lo hace en su extenso poema «Pórtico», publicado en 1892 como presentación del libro En tropel, de Salvador Rueda, y luego recogido, en 1895, en el poemario propio Prosas profanas. Como se comprueba en el análisis y comentario que hace Domínguez Caparrós en el centro exacto de la obra (pp. 8087), el poema tiene más de un centenar -113- de endecasílabos dactílicos acentuados en $1^{\mathrm{a}}, 4^{\mathrm{a}}$ y $7^{\mathrm{a}}$, lo que supone casi un $75 \%$ del total y constituye una clara y sistemática propuesta rítmica, apenas matizada por variaciones -fundamentalmente, y al lado de alguna excepción problemática pero a la vez anecdótica, la no acentuación de la primera sílaba- que no alteran lo esencial del 
carácter dactílico. Es en este poema, pues, donde el endecasílabo dactílico, merced a su notoria y rotunda presencia, se convierte en un verso de pleno derecho.

Sin embargo, y como exigen el orden cronológico y el rigor teórico, el estudio de Domínguez Caparrós comienza, tras unas breves notas preliminares, por la exposición y el análisis de los primeros testimonios teóricos -y la teoría implica sistema; es decir, las alusiones casuales y superficiales se dejan para un apartado posterior-sobre la existencia de este tipo de endecasílabos. El primer teórico recogido es Manuel Milá y Fontanals (pp. 8-12), que en 1875 se ocupa de este verso, aunque lo denomina anapéstico, no dactílico, debido, según se nos explica, a la analogía con un decasílabo anapéstico -típico de los himnos románticos y bien conocido en la época- al que se hubiera añadido una primera sílaba acentuada, y seguramente no computada al interpretarse como anacrusis. Milá, que identifica este verso con el endecasílabo de gaita gallega, de raíz popular, indaga su aparición en la poesía culta y moderna y señala como muestras algunos poemas de salón del s. XVII y versos de Iriarte, Masdeu, Moratín y Arnao.

Dejando a un lado menciones ocasionales, las de Milá y Fontanals son las únicas reflexiones teóricas anteriores a la aparición del mencionado poema de Darío. El siguiente teórico analizado, el chileno Eduardo de la Barra, no sólo da a la imprenta El endecasílabo dactílico con posterioridad a la publicación de «Pórtico», sino que lo hace precisamente con motivo de esa publicación $\mathrm{y}$, de manera más concreta, para intervenir en la controversia y defender la calidad y pertinencia del nuevo metro. Para ello, De la Barra reivindica su antigüedad, que según él se remontaría a la baja latinidad, y admite variantes casi análogas como la acentuación en la segunda sílaba, aunque defiende que el mejor que cabe encontrar en la modernidad es el de Rubén Darío, que renueva y dignifica el metro frente al uso imperfecto y descuidado que cabe encontrar en poetas como Iriarte o Moratín, y lo vuelve «una cadencia harmónica». En todo caso, De la Barra niega la oportunidad de denominarlo anapéstico, como habían hecho algunos estudiosos españoles, y desautoriza y rebate en distintos puntos al respecto a Eduardo Benot, Menéndez Pelayo o Leopoldo Alas Clarín. 
El siguiente autor cuyas reflexiones sobre el asunto consigna Domínguez Caparrós es el propio Clarín, que se siente obligado a terciar en la polémica ante las alusiones y críticas de De la Barra, al que responde apenas unos meses más tarde. La argumentación de Clarín es contundente en la forma y el contenido y contiene una notable dosis de ironía. Aparte de denunciar algunas inexactitudes de De la Barra, Clarín niega la pertinencia de hablar de endecasílabo dactílico en una métrica que carece de cantidad, y que se basa en el ritmo y las sílabas; el apellido, pues, «es pedantesco, inútil, contradictorio e incongruente», y asimilar acento y cantidad «es una metáfora arbitraria» y tal análisis se basa en un convencionalismo propio de una «métrica dedicada a sordomudos». El narrador identifica el verso descrito por De la Barra como el tradicional de gaita gallega, que en realidad es divisible, opina él, en hemistiquios, y, en definitiva -aunque con argumentos no siempre acertados, como el de que el endecasílabo no es múltiplo de tres, con lo que su acentuación no podría tener un carácter ternario-, atribuye este tipo de composición a ensayos defectuosos, tentativas frustradas en el período en el que el metro endecasilábico estaba en un proceso aún vacilante de formación, y luego, una vez que ya está consolidado el buen endecasílabo español, se trata simplemente de malos versos que rompen la armonía de los que cumplen la norma tradicional. Clarín critica - aunque no en la respuesta directa a Eduardo de la Barra- a Rubén Darío - «no es de mi parroquia»- y a Salvador Rueda por andar con novedades que no son sino «amaneramientos que muchas veces degeneran en notorios disparates».

El libro continúa con las ideas de Julio Vicuña Cifuentes (pp. 30-34), de quien destaca, en sus Estudios de métrica española, de 1929, la distinción de dos modalidades de endecasílabo «a minori», el que se acentúa en $4^{\mathrm{a}}$ y en $10^{\mathrm{a}}$-que daría la impresión de estar a medio hacer, de ser una especie de "sáfico frustrado»- y el que además se acentúa en $7^{\mathrm{a}}$, que Vicuña denomina endecasílabo siciliano, aunque también lo llama dactílico o de gaita gallega. Añade, además, nuevos ejemplos a los ya conocidos, aunque subraya que «es un verso hasta cierto punto casual», que en la poesía culta no constituye estrofa por sí mismo hasta Iriarte, y en él de manera fortuita, a diferencia de la versión 
popular, el endecasílabo de gaita gallega propiamente dicho, que se acentúa además en la primera sílaba.

El quinto autor tratado es Pedro Henríquez Ureña (pp. 34-36), que, como subraya Domínguez Caparrós, es el mayor conocedor de la versificación irregular hispánica y el responsable de la última monografía importante sobre el endecasílabo. Distingue en ella dos tipos principales, el endecasílabo acentuado en sexta y el acentuado en cuarta, aunque este último -el tipo B- se subdivide asimismo en tres especies: acentuado en $4^{\mathrm{a}}$ (B1), acentuado en $4^{\mathrm{a}}$ y $8^{\mathrm{a}}$ (B2) y acentuado en $4^{\mathrm{a}}$ y $7^{\mathrm{a}}$ (B3). Este último, en la poesía culta, sólo se mezclaría con los otros por inadvertencia. El endecasílabo anapéstico de la poesía popular, sin embargo, entraría con naturalidad en la versificación fluctuante de las canciones, «especialmente en las emparentadas con las de origen gallego». Por lo demás, Henríquez Ureña continúa añadiendo ejemplos a la nómina de autores que han utilizado el endecasílabo dactílico: Heredia, Martínez de la Rosa -en su traducción de la Romance Mauresque-, Perrús y Palacios, o, ya después de Darío, González Prada, Lugones, Mistral o Alberti, entre otros. Domínguez Caparrós destaca la importancia de la noticia que da Henríquez del poema de José María Heredia, titulado Himno de guerra, tanto por su notable extensión como por su temprana fecha de composición, 1826, aunque estuvo olvidado hasta su reedición en 1938.

Es Tomás Navarro Tomás el último tratadista destacado (pp. 36-39), que en su Métrica española de 1956 distingue la forma plena del endecasílabo dactílico - con acentos en $1^{\mathrm{a}}, 4^{\mathrm{a}}$ y $7^{\mathrm{a}}$ sílabas- y una manifestación parcial -acentuada en $4^{\mathrm{a}}$ y $7^{\mathrm{a}}-$. Navarro Tomás se suma a la consideración de que este verso surgiría a partir del endecasílabo sáfico «incompletamente acentuado», y sitúa su emancipación en una composición de siete cuartetos con rima aguda en los pares del primer tercio del s. XVIII, debida al mexicano fray Juan de la Anunciación y titulada «Al rayar la Aurora», aunque ya antes recuerda ejemplos insertados en diversas estrofas de la comedia Triunfos de amor y poder, del peruano Pedro Peralta Barnuevo. Tomás Navarro recoge los ejemplos citados por los tratadistas anteriores, y aporta algunos otros más como los de Sinibaldo de Mas, Santos Chocano, Lorca o Miguel 
Hernández, olvidando apenas, como subraya el autor, el caso de Benot, tal vez por no encontrarse en él valor poético que lo justifique. Como es norma en el libro de Navarro, ejemplos y autores se agrupan según la época, y se evidencia el período de esplendor que el endecasílabo dactílico conoce durante el modernismo, tras la intervención de Darío.

Antes de la conclusión de la parte teórica, hay un último apartado en esta primera sección del libro (pp. 39-50) en el que se recogen todas las referencias dispersas anteriores a Milá y Fontanals, con lo que la visión panorámica -panorámica por lo extenso, no precisamente por la falta de detalle, que es vicio ajeno por completo a esta obra y a su autor- queda completa y cerrada. Poetas como Iriarte y estudiosos como Coll y Vehí, Masdeu, Sinibaldo de Mas o Marroquín van haciendo del endecasílabo dactílico, progresivamente y bajo sus distintas denominaciones, un concepto más familiar. Domínguez Caparrós destaca por su importancia el caso de Andrés Bello; desde su Métrica de 1835 se reconoce su integración en la norma literaria moderna -merced al ejemplo de Iriarte-, así como su necesidad en un sistema silabotónico, y se impone el nombre de dactílico. Se da noticia, igualmente, del texto en el que Alberto Lista comenta que este verso es a veces un mal sáfico, y de cómo señala que los versos acentuados en séptima tal vez fueran agradables en composición independiente, pero nunca lo son «mezclados con los verdaderos endecasílabos», crítica que comparten, como se expone después, Quintana o Huerta.

El resto de la obra se dedica, como hemos dicho, al análisis de los principales ejemplos literarios que cabe encontrar en la poesía culta, con la mencionada subdivisión que marca la irrupción de Rubén Darío y su poema «Pórtico».

Los primeros casos - de los autores Joseph Blanch y José Bassa (pp. 55-56) - estarían vinculados directamente a la música; el tercero, de Pedro de Peralta (pp. 57-58), a la escena dramática -además, incluye algunos versos problemáticos-- Sólo a partir de fray Juan de la Anunciación (pp. 58-64) hay un endecasílabo dactílico claramente emancipado, que aparece en distintas composiciones suyas, algunas de las cuales recibieron el nombre de minué. No obstante, el carácter inédito de sus publicaciones 
hasta 1945 dejan a Tomás de Iriarte (pp. 65-67) como verdadero precursor del uso del endecasílabo dactílico en la tradición culta, y suele tomarse como primer ejemplo el de su fábula La criada y la escoba, que comienza con los versos:

Cierta criada la casa barría con una escoba muy puerca y muy vieja

Afirma Domínguez Caparrós que sólo el primer verso de la composición se ajusta fielmente al modelo del endecasílabo dactílico pleno -acentuado en $1^{\mathrm{a}}, 4^{\mathrm{a}}, 7^{\mathrm{a}}$ y $10^{\mathrm{a}}-$, y la sobreabundancia de acentuación en gran parte del resto desdibuja el ritmo al dificultar su percepción.

El siguiente poeta analizado es Leandro Fernández de Moratín (pp. 67-68), cuya importancia, matiza el autor, viene dada más por la repercusión en las teorías decimonónicas que por la calidad o el número de los ejemplos que proporciona. Tampoco serían del todo canónicos los endecasílabos de Heredia (pp. 6871), por su tendencia a acentuar la segunda sílaba, y los versos del siguiente escritor, Sinibaldo de Mas (pp. 71-72) -como los de Eduardo Benot (pp. 78-79)-, están aquejados de la artificialidad de quien compone un texto para ilustrar una teoría métrica propia, lo que les resta representatividad e incluso algo de legitimidad.

Los tres autores estudiados en los siguientes apartados están igualmente condicionados por diversos factores: Antonio Francisco de Castro (pp. 71-74) utiliza el metro en una traducción de Horacio -y emplea además numerosos acentos antirrítmicos-; Francisco Camprodón (pp. 74-76) y Antonio Arnau (pp, 76-77) comparten el hecho de que sus textos están vinculados a la música.

Después de todos estos poemas y canciones, que van prefigurando un metro que no acaba de consolidarse, objeto muchas veces de rechazo o al menos de polémicas, se llega por fin a la obra que lo reinventa, asienta y difunde, el poema «Pórtico», de Rubén Darío, que se convierte en la carta de naturaleza de este verso, en la referencia omnipresente y, en definitiva, y como su propio título augura, el portal por el que entra en la tradición 
literaria culta de manera más o menos oficial. Domínguez Caparrós da cuenta de algunos versos problemáticos o heterodoxos de este poema, y cita otros textos de Darío en que el endecasílabo dactílico tiene cierta presencia, pero no el mismo protagonismo; antes bien, nos recuerda que en distintos lugares de su obra lírica, contraviniendo conscientemente la preceptiva académica, recurre a la mezcla de este ritmo con otros endecasílabos, especialmente el pentámetro yámbico.

La última parte del libro, pues, se dedica a indagar en la fortuna que el endecasílabo dactílico corre después de la canonización que del mismo realiza Rubén Darío. La nota común, en este sentido, es una flexibilización heredada del poeta nicaragüense, que con su renovación trajo una acentuación variable, con la sola obligatoriedad de respetar los acentos en $4^{\mathrm{a}}$ y $7^{\mathrm{a}}$, en las que residiría la «esencia del modelo».

El primero de los poetas posteriores es, precisamente, aquel para cuyo poemario ofició de pórtico el poema de Darío, y que según algunos teóricos podría haber sido incluso antes modelo que epígono en el empleo del verso, por más que el de Darío tuviera más entidad y alcanzara una mayor repercusión. Siguen luego modernistas como González Prada (pp. 92-95), Antonio de Zayas (pp. 95-97), Eduardo Marquina (pp.100-108) -cuyo análisis es el más extenso del libro-, Leopoldo Lugones (pp. 119-121) o Francisco Villaespesa (pp. 112-113), junto a un ejemplo excepcional de Unamuno (pp. 126-127), el postmodernista Enríque Díez-Canedo (pp. 97-100) o el vanguardista Francisco Vighi (pp. 136-137). Amplían la nómina varios autores del Cono Sur que escriben en las primeras dos décadas del siglo XX -Enrique Banchs (pp. 108-112), Gabriela Mistral (pp. 113119) y Daniel de la Vega (pp. 120-121)-; autores relacionados con el grupo del 27 -Jorge Guillén (pp. 121-126), Rafael Alberti (pp. 131-133), Gerardo Diego (p. 135) y el más tardío Miguel Hernández (pp. 127-130)-, y otras voces de diversa procedencia y filiación como Eugenio Florit (p. 131), o Alfonso Reyes (pp. 133-134). Cierran la lista, como es lógico, las voces más recientes, las de Antonio Carvajal (pp. 137-139) y Pablo Jauralde Pou (pp. 139-140), y añade el autor como última nota que no es difícil encontrar ejemplos que, siguiendo la senda abierta por Rubén 
Darío, recurren a la inserción de algún endecasílabo dactílico entre endecasílabos de otros tipos, haciendo natural una combinación que la preceptiva ha prohibido durante largo tiempo.

La breve conclusión del libro (pp. 143-144) insiste en la división de la historia del endecasílabo dactílico en dos períodos, cuya línea divisoria sería la obra de Rubén Darío. Hasta él hubo ensayos asociados a la música o al ritmo de cláusulas, dentro de un ambiente «de búsqueda y ampliación de los metros hacia el silabotonismo», hasta convertirse en una necesidad teórica. La segunda etapa integra este ritmo en la métrica culta española con naturalidad y de una manera flexible, que incluso llega a combinarse con el endecasílabo yámbico.

La bibliografía (pp. 145-150), por último, es completa y rigurosa, y contiene todas las referencias pertinentes sobre el tema tratado, desde las más tempranas hasta las de las fechas más recientes, que serían el artículo de Mario Hernández de 2008 y el del propio José Domínguez Caparrós de 2009, publicado en la revista Rhythmica.

Se trata, en definitiva, de un estudio valioso que actualiza todo cuanto se ha dicho y se sabe acerca de este tipo de verso, probablemente el menos estudiado de los endecasílabos. El moderno endecasílabo dactílico, anapéstico o de gaita gallega viene a subsanar ese descuido general, y lo hace con gran profusión de datos y con la doble fundamentación de la perspectiva teórica, puramente métrica, y de la contextualización histórica que pone en relación la evolución del verso y de su uso con el devenir de las sucesivas poéticas, demorándose más en donde más lo demanda el asunto, especialmente en el momento clave de la irrupción de Rubén Darío y en la viva polémica consiguiente que envuelve a De la Barra y Clarín, y que afecta de manera directa a Salvador Rueda. Un valor añadido, por lo demás, de este libro es su voluntad de incluir todos aquellos textos que se mencionan y estudian, muchos de ellos en su totalidad, con lo que el análisis métrico, técnico y prolijo, alivia su necesaria frialdad con la vecindad de los poemas, pues es de literatura, al fin, de lo que se habla, y, como se ha dicho, esta obra no se limita a ser un imprescindible acercamiento al endecasílabo dactílico, a su historia y sus características métricas y rítmicas, sino que vale como meritoria anto- 
logía de autores muy dispares en cuanto a época y poética, pero que en algún momento de su trayectoria creadora comparten un rasgo formal tan marcado como significativo.

JuAN FraU

Universidad de Sevilla 